К.В. Грецких, А.С. Токарев

ГБУЗ «Научно-исследовательский институт скорой помощи им. Н. В. Склифосовского Департамента здравоохранения г. Москвы»; Россия, 129090 Москва, Большая Сухаревская пл., 3

К о н та к ты : Константин Валерьевич Грецких mail@rssklif.ru

\begin{abstract}
Злокачественные опухоли головного мозга считаются наиболее опасными не только из-за плохого прогноза, но и из-за непосредственного ухудшения качества жизни и когнитивных функций. Предполагается, что число таких больных будет увеличиваться по мере роста продолжительности жизни населения.

Летальность пациентов со злокачественными глиомами остается самой высокой среди пациентов с онкологическими заболеваниями. Медиана их выживаемости не превышает 24,5 мес. Несмотря на то что молекулярная биология серьезно продвинулась в изучении данного типа опухолей, остается открытым вопрос об эффективном применении этих знаний в лечебном процессе.

В обзоре освещены передовые методы диагностики и преимущества мультидисциплинарного подхода к лечению. Особое внимание уделено радиохирургическому лечению глиом высокой степени злокачественности, способному увеличить продолжительность жизни пациентов и улучшить ее качество.

Обзор разделен на 2 части. В 1-й части освещаются эпидемиология, клиника и диагностика глиом высокой степени злокачественности, а также комбинированный подход к их лечению.
\end{abstract}

Ключевые слова: стереотаксическая радиохирургия, гамма-нож, глиома высокой степени злокачественности, лучевая терапия, химиотерапия

Для цитирования: Грецких К.В., Токарев А.С. Глиомы высокой степени злокачественности: обзор литературы. Часть 1. Эпидемиология, классификация и подходы к комбинированному лечению. Нейрохирургия 2021; 23(1):124-34. DOI: 10.17650/1683-3295-2021-23-1-124-134.

\title{
High-grade gliomas: a literature review. Part 1. Epidemiology, classification and approaches to combination treatment
}

\section{K.V. Gretskikh, A.S. Tokarev}

N.F. Sklifosovsky Research Institute for Emergency Medicine, Moscow Healthcare Department; 3 Bolshaya Sukharevskaya Sq., Moscow 129090, Russia

\section{Cont a c t s : Konstantin Valerievich Gretskikh mail@rssklif.ru}

Malignant brain tumors remain one of the most complex problem in modern oncology, being among the most dangerous types of cancer not only because of their poor prognosis, but also due to the immediate consequences for quality of life and cognitive functions. It is expected that the number of such patients will increase as the life expectancy of the population increases.

The mortality rate of patients with malignant gliomas remains the highest among all cancer patients. The median survival rate in this population does not exceed 24.5 months. Despite serious progress in the study of the molecular biology of this type of tumor, the question of effective application of this knowledge in the treatment process remains open.

The review highlights the most advanced diagnostic methods and analyzes the effectiveness of a multidisciplinary therapeutic strategy. Special attention is given to the search for new approaches to radiosurgical treatment of highgrade gliomas in order to increase the duration and improve the quality of life of patients. 
The literature review is divided into 2 parts. Part 1 covers the epidemiology, clinic, and diagnosis of high-grade gliomas, as well as a combined approach to the treatment of the disease.

Key words: stereotactic radiosurgery, gamma knife, high-grade glioma, radiation therapy, chemotherapy

For citation: Gretskikh K.V., Tokarev A.S. High-grade gliomas: a literature review. Part 1. Epidemiology, classification and approaches to combination treatment. Neyrokhirurgiya = Russian Journal of Neurosurgery 2021;23(1):124-34. (In Russ.). DOI: 10.17650/1683-3295-2021-23-1-124-134.

\section{ВВЕДЕНИЕ}

Злокачественные глиомы головного мозга (ГМ) считаются самыми агрессивными первичными нейроэпителиальными опухолями центральной нервной системы (ЦНС) и характеризуются высокой смертностью и частотой локальных и отдаленных рецидивов [1-4].

Несмотря на то что молекулярная биология серьезно продвинулась в изучении данного типа опухолей, остается открытым вопрос об эффективном применении этих знаний в лечебном процессе $[5,6]$.

Широкое использование для решения этой проблемы самых передовых методов диагностики, обязательное применение мультидисциплинарного подхода (максимальная хирургическая резекция, лучевое воздействие на ложе опухоли, химиотерапия (XT)) не предотвращает неизбежное появление рецидива опухоли и не снижает почти абсолютную смертность [6-8]. Изучение данной проблемы осложняется высокой агрессивностью опухоли, ее склонностью к диффузному инфильтративному росту, невосприимчивостью к химическим агентам и лучевой терапии (ЛТ) $[9,10]$. Все перечисленное значительно затрудняет выработку тактики лечения [11]. Однако следует признать, что все чаще продолжительность жизни пациентов после комбинированного лечения стала превышать 1 год, и именно у таких пациентов наиболее часто возникают дистантные рецидивы глиом [12]. Все это требует нового подхода к лечению, способного увеличить продолжительность жизни и улучшить ее качество.

\section{1. ЭПИДЕМИОЛОГИЯ И ЭТИОЛОГИЯ}

Первичные опухоли ГМ в Международной классификации болезней 10-го пересмотра обозначены кодом C71. Они встречаются редко и составляют около $2 \%$ от общего числа случаев злокачественных новообразований. Наиболее распространенными вариантами первичных опухолей ГМ являются нейроэпителиальные опухоли, в группу которых входят глиомы высокой степени злокачественности (ГВС3) [13].

Злокачественные опухоли ГМ относятся к наиболее опасным онкологическим заболеваниям не только из-за плохого прогноза, но и из-за непосредственных последствий для качества жизни. Ожидается, что число пациентов будет увеличиваться по мере роста продолжительности жизни населения [18].

По данным Национального института рака (National Cancer Institute) США, в 2007 г. заболеваемость первич- ными опухолями ЦНС в США составила 6,6 случая на 100 тыс. человек, а ожидаемая смертность 4,22 случая на 100 тыс. [14].

По официальным статистическим данным, в Российской Федерации заболеваемость первичными опухолями ЦНС в 2010 г. составила 4,2 случая на 100 тыс. человек, в 2015 г. - 4,8 случая. Российский центр информационных технологий и эпидемиологических исследований в области онкологии Московского научно-исследовательского онкологического института им. П.А. Герцена сообщил, что абсолютное число впервые установленных диагнозов злокачественных новообразований ГМ и других отделов ЦНС (С70 С72) в 2015 г. достигло 4377. Средний возраст пациентов с впервые установленным диагнозом злокачественного новообразования ЦНС (C71 - C72) в России в 2015 г. равняется 53,5 года (в 2005 г. - 48,7 года). Общая заболеваемость опухолями данной локализации в России в 2015 г. составила 8896 случаев, в том числе 655 - среди детей (в возрасте до 17 лет) [15].

Глиомы возникают из клеток глии, входящих в паренхиму мозга. На их долю в структуре первичных опухолей ГМ приходится 15,6 \%. В этой группе новообразований глиобластома является самой распространенной первичной злокачественной опухолью ГМ у взрослых [16].

Несмотря на то что глиомы могут развиться в любом возрасте, как правило, они обнаруживаются у больных 30-60 лет [17-19], т. е. эти опухоли поражают наиболее трудоспособную часть населения. У мужчин риск развития глиом выше, чем у женщин, в 1,5 раза, а у пожилых людей выше, чем у молодых, в 3,2 раза [16].

Медиана выживаемости большинства пациентов с глиобластомой с момента постановки диагноза не превышает 15 мес, при этом 2-летняя выживаемость составляет 26-33\% [20].

Единственная достоверно известная причина возникновения опухолей ГМ - воздействие радиации [17]. Высказываются также предположения о том, что этиологическими факторами могут быть винилхлорид, аспартам (заменитель сахара) и электромагнитные поля мобильных телефонов или линий высоковольтных передач [21]. Существует и ряд наследственных синдромов, в клинической картине которых могут встречаться ГВСЗ: нейрофиброматоз I и II типов, синдром Ли-Фраумени, туберозный склероз, болезнь Олье и синдром Маффуччи, синдром Линча и др. [22]. 


\section{2. МОЛЕКУЛЯРНО-ГЕНЕТИЧЕСКАЯ}

ХАРАКТЕРИСТИКА И КЛАССИФИКАЦИИ

Первичный диагноз верифицируют с помощью иммуногистохимического (ИГХ) исследования, в ходе которого выявляют в клетках глиомы глиальный фибриллярный кислый белок (glial fibrillary acidic protein), который является основным промежуточным филаментным белком зрелых астроцитов [23]. Повышение злокачественности опухолей астроцитарного происхождения связано с утратой экспрессии этого белка [24].

В классификации Всемирной организации здравоохранения (ВО3) подтипы ГВСЗ выделяются на основании их гистологического и ИГХ сходства с предполагаемыми клетками происхождения. Градация проводится согласно гистологическим признакам биологической агрессивности опухоли: количеству некрозов, митотическому индексу и степени гиперплазии эндотелия сосудов [25].

Глиобластомы принято подразделять на первичные и вторичные. Первичные глиобластомы возникают de novo без каких-либо признаков предшествующего поражения. Вторичные глиобластомы развиваются из ранее существовавших астроцитом низкой степени злокачественности [26]. Типичные изменения, характерные для первичной глиобластомы, - мутация и амплификация гена $E G F R$ (epidermal growth factor receptor, рецептор эпидермального фактора роста), делеция $P 16$ и утрата гетерозиготности на длинном плече 10-й хромосомы, содержашей PTEN (phosphatase and tensin homolog, гомолог фосфатазы и тензина). Визитная карточка вторичной глиобластомы - избыточная экспрессия PDGFA/PDGFR $\alpha$ (platelet-derived growth factor ligand $\mathrm{A} /$ platelet-derived growth factor receptor $\alpha$, лиганд А фактора роста тромбоцитов и его рецептор $\alpha$-типа), утрата гетерозиготности на длинном плече 19-й хромосомы и мутации IDH1/2 (isocitrate dehydrogenase $1 / 2$, изоцитратдегидрогеназа 1 и 2), TP53, $\operatorname{ATRX}[25,27,28]$.

Отметим, что если мнение о природе опухоли и степени малигнизации совпадает у 3 специалистовпатоморфологов, то формулируется диагноз, включающий название опухоли с указанием степени злокачественности: grade I-II по ВО3 при доброкачественных опухолях и grade III-IV при злокачественных (это стандарт диагностики). В некоторых случаях необходимо ИГХ исследование опухоли с определением индекса Ki-67 для уточнения степени малигнизации опухоли (это рекомендация) [29, 30]. Прогноз при опухолях III степени злокачественности лучше, чем при опухолях IV степени, но они также прогрессируют и характеризуются аналогичным клиническим течением.

Прогностическое значение имеют мутации генов, кодирующих IDH1/2, которые играют важную роль в метаболизме глюкозы [31]. Метилирование гена $M G M T$ (O-6-methylguanine-DNA methyltransferase, O-6-мe-
тилгуанин-ДНК-метилтрансфераза) ассоциировано с чувствительностью к ХТ и ЛТ [29].

При олигодендроглиальных опухолях коделеция или утрата гетерозиготности короткого плеча 1-й хромосомы и длинного плеча 19-й хромосомы указывает на лучший прогноз (при опухолях III степени злокачественности по ВО3) [29].

Малигнизация глиом низкой степени злокачественности продолжается от 4 до 29 мес. Примерно у $45 \%$ пациентов диффузные низкодифференцированные глиомы II степени злокачественности по ВО3 трансформируются в анапластические глиомы (grade III) в течение 5 лет [32].

Классификация ВО3 является действующим международным стандартом номенклатуры и диагностики глиом. Она была впервые опубликована в 1979 г. и пересмотрена с тех пор 4 раза, последний раз в 2016 г. В основу современных классификаций опухолей группы глиом по гистогенетическим признакам легла классификация, опубликованная в 1926 г.; она выделяла 3 степени злокачественности [33].

По классификации ВОЗ глиомы, которые имеют низкий пролиферативный потенциал и могут быть излечены хирургическим путем, относятся к поражениям I степени злокачественности. Глиомы II степени относительно незлокачественные: они также имеют слабую склонность к росту и распространению, но способны рецидивировать после лечения. Глиомы III-IV степени являются высокозлокачественными и инвазивными. IV степень присваивают глиобластомам ввиду их максимальной агрессивности и инвазивности; для нее типичны участки некрозов и обширные поля de поvо образованных сосудов [33].

Согласно последнему изданию классификации ВО3 глиобластомы должны быть определены с обязательным указанием на наличие или отсутствие мутаций гена $I D H$ : мутированный или «дикий тип». Для разграничения диффузных глиом и олигодендроглиом в классификации использован новый критерий - наличие или отсутствие мутаций генов $I D H$ и $A T R X$ на фоне отсутствия или наличия коделеции $1 \mathrm{p} 19 \mathrm{q}[34]$.

Следует упомянуть о системе оценки St. AnneMayo, также известной как система оценки DaumasDuport (введена в 1988 г.). Она некоторое время была популярна при оценке диффузных астроцитом, но сейчас практически полностью заменена классификацией ВO3.

Пациенты с диагнозом ГВСЗ имеют одинаково плохой прогноз, с медианой выживаемости после рецидива 39-47 нед при III степени злокачественности и 25-30 нед при IV степени [35, 36]. Традиционные прогностические факторы выживаемости включают возраст, работоспособность, гистологические признаки, тяжесть симптомов и степень полноты резекции [37]. 


\section{3. КЛИНИЧЕСКАЯ КАРТИНА}

\section{И ДИАГНОСТИКА}

Признаки рецидива ГВСЗ очень разнородны и определяются как расположением и распространенностью новообразования, так и степенью выраженности перифокального отека.

Прямое воздействие, при котором мозговая ткань разрушается в результате некроза, приводит к возникновению общемозговой (головные боли, ликворногипертензионный синдром) и очаговой неврологической симптоматики (симптомы выпадения/снижения функции движения, нарушение функций черепных нервов, чувствительности, выпадение высших корковых функций, бульбарные и вестибулярные расстройства).

В финальной стадии заболевания и в целом при рецидивировании преобладает дислокационная симптоматика.

Нередко рецидив опухоли манифестирует судорожным синдромом. В зависимости от локализации опухоли в 20-40 \% случаев также могут наблюдаться судорожные припадки, обычно с очаговым началом, которые могут быть простыми частичными, сложными частичными или генерализованными $[13,14,38]$.

К опосредованным эффектам относится повышенное внутричерепное давление вследствие постепенного увеличения размеров опухоли и усиления отека вокруг нее, что проявляется головными болями у $30-50 \%$ пациентов с глиобластомами [39].

Методы нейровизуализации, которыми мы располагаем на сегодняшний день, позволяют получить достоверную информацию о расположении, распространенности, вероятной гистоструктуре опухоли и наличии перифокального отека.

У лиц с подозрением на ГВСЗ применяют инвазивные (катетерная ангиография) и неинвазивные (компьютерная (KT) и магнитно-резонансная томография (МРТ)) методы диагностики. Для выявления ранних послеоперационных осложнений проводят нативную и контрастную КТ и МРТ; для исключения рецидива глиомы требуется сочетание МРТ (включая перфузионные последовательности) и позитронной эмиссионной томографии с мечеными аминокислотами (чаще всего метионином или тирозином) [41, 42].

При КТ ГВСЗ обычно визуализируются как гипоинтенсивные участки, вызывающие смещение срединных структур ГМ вследствие перифокального отека.

И все же стандартом нейровизуализации считается МРТ, выполненная в Т1- и Т2-взвешенных последовательностях, которая позволяет с высокой точностью заподозрить ГВСЗ, что обусловлено отличным контрастированием мягких тканей, визуализацией неоднородности опухолевого поражения и анатомо-топографических особенностей новообразования [40]. Результаты МРТ, усиленной гадолиниевым контрастом, позволяют точно выявить центральную область некроза, область накопления контрастного вещества и зону отека белого вещества $[14,18]$.

ГВСЗ почти всегда выглядят как очаг гетерогенного усиления накопления гадолиния на Т1-взвешенных изображениях; при анапластических глиомах усиление более вариабельно. Наиболее полезными для полной визуализации отека, ассоциированного с ГВСЗ, являются Т2-взвешенные изображения с подавлением сигнала от жидкости (FLAIR) [41, 42].

Ряд авторов считает, что при расположении опухоли в функционально значимых областях для верификации расположения речевых, чувствительных и двигательных зон необходимо выполнение МРТ-трактографии и функциональной МРТ [43, 44]. С их точки зрения, применение этих методик позволяет картировать функционально значимые зоны мозга и свести к минимуму риск возникновения неврологического дефицита в послеоперационном периоде [45]. При определении объема лечения следует также учитывать потенциальное распространение глиомы по срединной линии через мозолистое тело («симптом бабочки») [40].

Многие авторы полагают, что важным и информативным дополнением при визуализации ГВСЗ могут быть позитронная эмиссионная томография с мечеными аминокислотами [41], однофотонная эмиссионная KT [46], транскраниальная магнитная стимуляция [47].

L. Nayak, D.A. Reardon считают, что окончательный диагноз возможно поставить только после исследования удаленной опухоли или ее частей, взятых при биопсии, с использованием гистологических, цитологических и ИГХ методов [36].

О.В. Абсалямова и др. отмечают, что истинная зона распространения ГВСЗ обширнее, чем часть опухоли, визуализируемая при любом виде томографии [21]. Этот инфильтративный тип роста в значительной степени препятствует точному определению границы между опухолью, перифокальным отеком и интактной частью мозга, что снижает радикальность лечения [48].

Многие авторы считают полезной перфузионную МРТ (функциональную нейронавигацию) как на этапе планирования операции, так и во время ее проведения для оценки васкуляризации опухоли и проницаемости эндотелия, а также диффузионно-тензорную трактографию для отображения подкорковых трактов белого вещества, окружающих глиому [29, 40, 42].

Таким образом, использование данных МРТ и КТ для планирования лечения в настоящее время рассматривается как стандарт оказания медицинской помощи при ГВС3 [29, 40].

\section{4. ХИРУРГИЧЕСКОЕ ЛЕЧЕНИЕ}

Резекция играет главную роль в лечении ГВСЗ. Однако инфильтративная природа глиом, их склонность к микроскопическому рассеянию в мозге по-прежнему представляют собой серьезное препятствие для полной резекции. 
Y.R. Lawrence и соавт. полагают, что даже при четко очерченном поражении МРТ обычно недооценивает распространение инфильтрирующих глиом. Опухолевые клетки обнаруживаются на расстоянии до 20 мм от визуализированных границ ГВСЗ, а также во всем вовлеченном полушарии и даже в контралатеральном [49]. Как результат, рецидив опухоли часто возникает в непосредственной близости от пострезекционной полости [50].

Хирургическое лечение опухолей ГМ выполняет 3 функции: постановка гистологического диагноза, улучшение состояния больного (благодаря быстрому уменьшению объема опухоли) и прогноза. Если резекция невозможна, необходимо провести биопсию для получения образца ткани и постановки окончательного диагноза [29].

Следует признать, что хирургическое лечение может привести к излечению только в случае глиом I степени злокачественности по ВО3, а при опухолях IIIV степени злокачественности получить подобный результат в настоящее время нельзя. Глиомы II и III степени злокачественности в конечном итоге претерпевают трансформацию в опухоли более высокой злокачественности, и поэтому хирургическое удаление в сочетании с химиолучевой терапией позволяет только замедлить прогрессирование и в некоторой мере продлить жизнь.

Растет количество доказательств повышения общей выживаемости и выживаемости без прогрессирования и улучшения качества жизни по мере увеличения объема резекции [29].

В многочисленных исследованиях продемонстрировано, что опухолевые клетки могут быть обнаружены (хотя и с меньшей частотой) в участках, отдаленных от первичного очага, что указывает на то, что «полной» резекции опухоли может быть недостаточно [51]. Несмотря на это, доказано, что почти полная резекция опухоли (>98 \% объема), после которой остается не более 1-2 см ${ }^{3}$ опухоли, может значительно продлить жизнь пациентов [52-54]. При глиомах низкой степени злокачественности 10-летняя выживаемость больных составляет $91 \%$, если объем резекции превышает $90 \%$. У пациентов с ГВСЗ также наблюдается улучшение выживаемости и качества жизни при максимальной резекции [29].

В то же время всегда следует помнить, что стремление получить оптимальный онкологический результат ограничено вероятностью выпадения неврологических функций после радикального иссечения тканей ГМ [55].

Остаточный объем опухоли определяется как область накопления контрастного препарата, выявленная при послеоперационной МРТ. В ряде ретроспективных исследований подтверждено, что меньший остаточный объем ассоциирован с более высокой выживаемостью без прогрессирования и общей выжива- емостью $[54,56]$. В частности, определен максимальный остаточный объем, ассоциированный с высокой выживаемостью при ГВСЗ; он составил 5 cм $^{3}$ [56]. Помимо этого остаточный объем, накапливающий контрастное вещество, и остаточный объем, выявленный по соотношению T2/FLAIR, могут быть независимыми предикторами выживаемости в дополнение к расширенному объему резекции [50].

Кроме того, высказано предположение, что объем резекции $\geq 80 \%$ является минимумом, обеспечивающим преимущество в выживаемости при рецидивирующей глиобластоме [3].

Хирургическое вмешательство по-прежнему остается первым шагом в лечении пациентов с подозрением на ГВСЗ. Биопсию выполняют при мультифокальном поражении или при высоком риске серьезной инвалидизации пациентов после резекции опухоли [57].

Следует отметить, что такие интраоперационные технологии, как картирование функционально значимых зон ГМ, кортикальная и подкорковая стимуляция, МРТ, функциональная, ультразвуковая метаболическая нейронавигация, используются для идентификации границ опухоли и сохранения важных структур ГМ [50].

По данным ряда авторов, предикторами исхода заболевания следует считать также возраст пациента, гистологические особенности опухоли, индекс Karnofsky и некоторые молекулярные маркеры (коделецию $1 \mathrm{p} 19 \mathrm{q}$, статус $I D H$, статус метилирования промотора MGMT, мутация $A T R X)$ [29, 31, 52].

R.S. D'Amico и соавт. полагают, что целью нейрохирурга должен оставаться оптимальный баланс между максимально полным удалением опухоли и сохранением неврологических функций [50]. Поддержание качества жизни после резекции глиомы является критически важным и оказывает существенное влияние на выживаемость.

\section{5. ХИМИОТЕРАПИЯ}

Исторически сложилось так, что опухоли ЦНС трудно поддавались стандартной хирургической резекции, ЛТ и ХТ. Однако за последние десятилетия наметился прогресс благодаря пониманию молекулярных процессов, протекающих в глиомах со злокачественным фенотипом. Это привело к появлению нескольких новых химиотерапевтических подходов.

A.P. Kyritsis, V.A. Levin указывают, что отсутствует единое мнение о том, существуют ли иные варианты ХТ при рецидивирующем или прогрессирующем течении заболевания после отказа от темозоломида. Причинами низкой эффективности препаратов являются низкая проницаемость для них гематоэнцефалического барьера, продукция белков множественной лекарственной устойчивости в клетках глиомы и ассоциированных с ней капиллярах [58], генетическая и метаболическая гетерогенность клеток глиомы, peзистентность к алкилирующим агентам [59]. 
Стволовые клетки глиомы могут провоцировать ее развитие, обеспечивать устойчивость к ХТ и ЛТ, что обусловливает неэффективность стандартных методов лечения и возникновение рецидивов [60]. Кроме того, ЛТ, являющаяся основным методом лечения глиом, запускает несколько путей передачи сигналов и токсических событий в опухоли, таких как прямое повреждение ДНК. Это может привести либо к гибели клеток, либо к мутациям в выживших опухолевых клетках, которые могут способствовать появлению резистентных клонов и рецидивам [59].

Послеоперационная адъювантная ЛТ и ХТ монофункциональным алкилирующим агентом 2-го поколения темозоломидом стала стандартным методом 1-й линии лечения впервые диагностированных злокачественных глиом [20, 59, 61].

Темозоломид - это алкилирующий цитостатик, который имеет тот же активный метаболит, что и дакарбазин. Это относительно небольшая молекула (194 Да), и она легко проникает через гематоэнцефалический барьер благодаря своей липофильности. Стандартная схема монотерапии темозоломидом: 150-200 мг/м² в течение 1-5 дней каждые 4 нед [59].

К сожалению, ГВСЗ обычно рецидивируют, несмотря на лечение темозоломидом, что требует решения вопроса о необходимости повторной операции. Однако для больных с рецидивирующей глиомой, не проходивших адъювантной терапии темозоломидом, этот вариант лечения по-прежнему остается хорошим выбором [58].

Стандартное лечение впервые диагностированной глиобластомы после хирургической резекции - ЛТ (60 Гр в 18-20 фракциях) с одновременным ежедневным приемом темозоломида и последующие 6 или более циклов адъювантной терапии темозоломидом [3, 58].

Проспективное рандомизированное исследование 573 пациентов в возрасте $\leq 70$ лет с впервые диагностированной глиобластомой показало, что после ЛТ с непрерывным ежедневным приемом темозоломида (75 мг/м²/сут) и последующей 6-месячной адъювантной терапии темозоломидом (150-200 мг/м²/сут в течение 5 дней 28-дневного цикла) медиана выживаемости была больше (14,6 мес), чем после ЛТ без терапии темозоломидом (12,1 мес). Что еще более важно, 2-летняя выживаемость также была выше - соответственно 26,5 и $10,4 \%[62,63]$.

В нескольких исследованиях с целью улучшения выживаемости пациентов были протестированы несколько алкилирующих агентов 2-й линии ХТ глиобластом: кармустин (BCNU, 1,3-бис (2-хлорэтил) 1-нитрозомочевина) и ломустин (CCNU, 1-(2-хлорэтил)-3-циклогексил-1-нитрозомочевина) [63, 64]. Кармустин и ломустин оказались резко цитотоксичными и приводили к раннему развитию резистентности и многим побочным эффектам, что ограничило их применение [64, 65].
Требует дальнейшего изучения применение винкристина [66] и глиадела [67] при рецидиве ГВС3 из-за опасений по поводу высокого риска побочных эффектов и сомнительной эффективности.

\section{6. ТАРГЕТНАЯ ТЕРАПИЯ}

Большинство авторов полагает, что только ту терапию, которая воздействует на конкретные сигнальные пути, будь то рецепторы клеточной поверхности, сигнальные белки в цитозоле или ферменты, важные для транскрипции ДНК в ядре, можно назвать таргетной терапией [68-74]. Но многие из новых методов таргетного лечения нацелены на 3-5 или более белков и поэтому далеки от того, чтобы считаться специфичными [58].

По мнению C. Izquierdo и соавт., доклинические и клинические данные свидетельствуют о том, что комбинированное ингибирование серин-/треониновой протеинкиназы B-raf (BRAF) и митогенактивированных протеинкиназных ферментов (МЕК) может быть более эффективным, чем ингибирование только BRAF [68].

C.J. Wheeler указывает, что в качестве дополнения к стандартным методам лечения ГВСЗ могут быть использованы репликационно-некомпетентные или компетентные вирусы в качестве векторов либо в качестве средств индуцирования онколиза с предотвращением повреждения соседних нормальных клеток [69].

J.A. Carrillo и соавт. отмечают, что углубление знаний о молекулярных подтипах глиом и лежащих в их основе изменениях в специфических сигнальных путях до сих пор не привело к успеху таргетной терапии, как это имело место в случае других солидных опухолей. Сегодня единственным препаратом для таргетной терапии глиом, одобренным Управлением по санитарному надзору за качеством пищевых продуктов и медикаментов (Food and Drug Administration) США, является бевацизумаб, который воздействует на фактор роста эндотелия сосудов (vascular endothelial growth factor), представляющий собой потенциально перспективную мишень [70].

Бевацизумаб - гуманизированное (приближенное к человеческому) моноклональное антитело, которое инактивирует циркулирующий VEGF-A и изменяет его связывание с эндотелиальными клетками, тем самым блокируя ангиогенез [70]. При этом предотвращается образование новых кровеносных сосудов, что крайне важно при рецидивирующей глиобластоме [7, 13].

Такие антиангиогенные агенты, как седираниб (ингибитор тирозинкиназы рецептора VEGF) и циленгитид (ингибитор интегрина), в исследованиях III фазы не изменяли исход [72, 73].

Продолжается рандомизированное проспективное клиническое исследование (RTOG 1205) эффективности одновременного применения бевацизумаба и облучения в сравнении с изолированным применением 
бевацизумаба при рецидивирующих глиобластомах [24, 75].

По мнению М. Ameratunga и соавт., антиангиогенная терапия стала основой лечения рецидивов ГВС3, несмотря на отсутствие достаточных клинических доказательств ее эффективности [72]. Ретроспективный анализ исследования показал, что люди с пронейральной глиобластомой с диким типом IDH1 получают преимущество в выживаемости от первичной терапии бевацизумабом, однако это не было подтверждено на независимом наборе данных [76].

Исследование CABARET показало, что в обеих группах лечения, получавших бевацизумаб, качество жизни улучшилось у одинаковой доли участников. Тем не менее бевацизумаб как самостоятельно, так и в комбинации с XТ, не превосходил ХТ по эффективности или качеству жизни [77].

На данном этапе влияние бевацизумаба на качество жизни и исход остается неясным [72]. Однако, по мнению О.С. Andronesi и соавт., при рецидивирующей ГВС3 у пациентов с метилированным MGMT следует применять только бевацизумаб, а затем 13-цис-ретиноевую кислоту (cRA). У пациентов с неметилированным MGMT (вероятно, резистентным к алкилирующим агентам) можно было бы использовать бевацизумаб или иринотекан с последующим назначением сRA [78].

Последовательный анализ мутаций в первичной глиоме и каждом последующем рецидиве позволил предположить, что хирургическое вмешательство и химиолучевая терапия заставляют опухоль выбирать альтернативные пути злокачественного прогрессирования $[12,79]$.

В связи с тем, что мутации в генах IDH1/2 занимают центральное место в онкогенезе глиом II и III степени злокачественности, в настоящее время проводятся исследования вакцин против IDH1, малых молекул - ингибиторов IDH1 и IDH2, а также метаболических компонентов, включая истощение $\mathrm{NAD}+$, нацеленных на глиомы с мутацией $I D H[79,80]$.

К методам терапевтического воздействия на ГВС3, которые не отражены в данном обзоре, но интенсивно изучаются, относятся лазерная интерстициальная термическая терапия, иммунотерапия, онколитическая вирусотерапия, конвективно-усиленная доставка, высокоинтенсивная фокусированная ультразвуковая абляция, ТTF-терапия и применение наночастиц. Исследуемые препараты - ингибиторы контрольных точек, вакцины против опухолеассоциированных антигенов и опухолеспецифических антигенов, импульсные дендритные клетки и CAR-T-клетки [71] и др.

\section{7. ЛУЧЕВАЯ ТЕРАПИЯ}

Метод ЛТ основан на эффектах, возникающих при прохождении ионизирующего излучения сквозь ткани организма: фотоэффекте, эффекте Комптона и эффекте образования пар. Подобное воздействие приводит к ионизации вещества и появлению радиобиологического ответа, который подробно описан в соответствующих работах [81].

Высокая эффективность ЛТ при лечении злокачественных новообразований объясняется известным правилом, сформулированным в 1906 г. французскими учеными J. Bergonie и L. Tribondeau: в живой ткани клетки тем более радиочувствительны, чем менее они дифференцированы, чем больше выражена их пролиферативная активность и продолжительнее у них процесс митоза [82].

Стандартное лечение глиобластомы включает фракционированное облучение в суммарной дозе 60 Гр, которая делится на 30-33 фракции, доставляемые в течение примерно 6 нед. К общему объему облучаемой опухоли добавляют запас в 2-3 см для облучения ее инфильтрирующей части [81]. Лучевая терапия была первым методом лечения, чья эффективность у пациентов с ГВСЗ была доказана в рандомизированных исследованиях. Послеоперационная ЛТ улучшила медиану ОВ пациентов с глиобластомами с 3-5 до 8-10 мес [81].

Хирургическая резекция обычно ограничивается участком глиобластомы, который накапливает гадолиний. Оставшаяся рядом инфильтрированная опухолевыми клетками мозговая ткань обрабатывается комбинацией ЛТ и XТ [74, 81]. Стандартным режимом послеоперационной терапии для пациентов с впервые диагностированной глиобластомой в хорошем общем состоянии является ЛТ в сочетании с ежедневным приемом темозоломида в дозе 75 мг/ м $^{2}$ с последующим интервалом примерно 4 нед без терапии и дальнейшим проведением до 6 циклов терапии темозоломидом в дозе 150-200 мг/м² в течение 5 дней (цикл длительностью 28 дней) [60, 83].

В ряде исследований изучена эффективность доз, превышающих 60 Гр, в лечении глиобластом различными методами ЛТ, включая 3D-конформную ЛТ, стереотаксическую радиохирургию, брахитерапию. Однако даже при дозах, достигающих 90 Гр, возникал рецидив опухоли, и повышение дозы в ходе брахитерапии не увеличило выживаемость в рандомизированном исследовании. Радиационный некроз также часто оказывается лимитирующим фактором, препятствующим увеличению дозы $[60,84]$.

В исследовании A. Sourati с учетом плохих исходов после стандартной ЛТ и недостаточной ее эффективности даже при увеличении дозы было изучено несколько других подходов, включая гиперфракционирование, но доказательства улучшения выживаемости не получены [84].

В настоящее время режимы гипофракционирования достаточно широко распространены и внедрены в практическую радиологию, в основном при рецидивах заболевания. По мнению G.M. Clark и соавт., курс 
ЛТ из 5 фракций по 7 Гр у пациентов с рецидивом ГВСЗ позволяет поддерживать удовлетворительное качество жизни в приемлемые сроки, сопоставимые с результатами ХТ [75].

Необходимо отметить, что с целью повышения эффективности ЛТ ведутся работы по поиску эффективных радиосенсибилизаторов - препаратов, синергически взаимодействующих с ионизирующей радиацией. Для этого системную терапию часто проводят одновременно с ЛТ или с применением других повреждающих ДНК агентов для усиления местного контроля за ГВСЗ. С этой целью темозоломид назначают в качестве радиосенсибилизатора в рамках действующего стандарта оказания медицинской помощи после хирургической резекции глиобластомы [14, 21, 85].

Потенциальные острые токсические реакции после ЛТ включают усталость, эритему кожи, головную боль, алопецию, тошноту, реже судороги. Долгосроч- ные последствия облучения - постоянная усталость, катаракта, потеря зрения, слуха, ксеростомия, лейкоэнцефалопатия, эндокринопатия и нейрокогнитивные нарушения.

Вторичный (вызванный ЛТ) некроз головного мозга является дозозависимым и может потребовать применения кортикостероидов или хирургического вмешательства (при наличии тяжелых симптомов).

По мнению ряда авторов, риск развития радиационно-ассоциированных вторичных злокачественных новообразований через 20 лет после облучения ЦНС составляет 1-3 \%; обычно это наблюдается у тех, кто прошел ЛТ в детском или молодом возрасте [83].

До появления КТ и МРТ облучение всего мозга обычно использовали в качестве адъювантной ЛТ при ГВС3 [86]. На современном этапе по мере развития планирования ЛТ на основе МРТ-изображений стало возможным более локальное облучение.

\section{Л И TE P A I PA I REFERE N GES}

1. Thakkar J.P., Dolecek T.A., Horbinski C. et al. Epidemiologic and molecular prognostic review of glioblastoma. Cancer Epidemiol Biomarkers Prev 2014;23(10):1985-96. DOI: 10.1158/1055-9965.epi-14-0275.

2. Бельский К.К., Гуров Д.Ю., Колесников А.Е. Заболеваемость злокачественными глиомами головного мозга в Волгоградской области. Российский онкологический журнал 2010;(4): 39-42. [Belskiy K.K., Gurov D.Yu., Kolesnikov A.E. The incidence of malignant gliomas of the brain in the Volgograd region. Rossijskiy onkologicheskiy zhurnal = Russian Journal of Oncology 2010;(4):39-42. (In Russ.)].

3. Gilbert M.R., Wang M., Aldape K.D. et al. Dose-dense temozolomide for newly diagnosed glioblastoma: a randomized phase III clinical trial. J Clin Oncol 2013;31(32):4085-91. DOI: $10.1200 /$ jco. 2013.49 .6968

4. Ostrom Q.T., Gittleman H., Farah P. et al. CBTRUS statistical report: primary brain and central nervous system tumors diagnosed in the United States in 20062010. Neuro Oncol 2013;15 Suppl 2:ii1-56. DOI: $10.1093 /$ neuonc/not 151 .

5. Malignant Brain Tumors. Ed. by J. Moliterno Gunel, J.M. Piepmeier, J.M. Baehring. New York: Springer, 2017. DOI: 10.1007/978-3-319-49864-5.

6. Chen J., McKay R.M., Parada L.F. Malignant glioma: lessons from genomics, mouse models, and stem cells. Cell 2012;149(1):36-47.

DOI: 10.1016/j.cell.2012.03.009.

7. Chamberlain M.C. The paradoxical effect of bevacizumab in the therapy of malignant gliomas. Neurology 2011;77(8):803-4.

DOI: 10.1212/wnl.0b013e3182247068.

8. Cheon Y.J., Jung T.Y., Jung S. et al. Efficacy of Gamma Knife radiosurgery for recurrent high-grade gliomas with limited tumor volume. J Korean Neurosurg Soc 2018;61(4):516-24. DOI: $10.3340 /$ jkns.2017.0259.

9. Oppenlander M.E., Wolf A.B., Snyder L.A. et al. An extent of resection threshold for recurrent glioblastoma and its risk for neurological morbidity. J Neurosurg 2014;120(4):846-53. DOI: $10.3171 / 2013.12$.jns13184.

10. Hong B., Wiese B., Bremer M. et al. Multiple microsurgical resections for repeated recurrence of glioblastoma multiforme. Am J Clin Oncol 2013;36(3):261-8. DOI: 10.1097/COC.0b013e3182467bb1.

11. Park J.K., Hodges T., Arko L. et al. Scale to predict survival after surgery for recurrent glioblastoma multiforme. J Clin Oncol 2010;28(24):3838-43. DOI: $10.1200 / J C O .2010 .30 .0582$.

12. Mazor T., Pankov A., Johnson B.E. et al. DNA methylation and somatic mutations converge on the cell cycle and define similar evolutionary histories in brain tumors. Cancer Cell 2015;28(3):307-17. DOI: 10.1016/j.ccell.2015.07.012.

13. Zikou A., Sioka C., Alexiou G.A. et al. Radiation necrosis, pseudoprogression, pseudoresponse, and tumor recurrence: imaging challenges for the evaluation of treated gliomas. Contrast Media Mol Imaging 2018;2018:6828396. DOI: $10.1155 / 2018 / 6828396$.

14. Omuro A., DeAngelis L.M. Glioblastoma and other malignant gliomas: a clinical review. JAMA 2013;310(17):1842-50. DOI: 10.1001/jama.2013.280319.

15. Злокачественные новообразования в России в 2017 году (заболеваемость и смертность). Под ред. А.Д. Каприна, В.В. Старинского, Г.В. Петровой. М.: МНИОИ им. П.А. Герцена, 2018. 250 с. [Malignant tumors in Russia in 2017 (morbidity and mortality). Ed by A.D. Kaprin, V.V. Starinsky, G.V. Petrova. Moscow: MNIOI im. P.A. Gertzena, 2018. 250 p. (In Russ.)].

16. Трашков А.П., Спирин А.Л., Цыган Н.В. и др. Глиальные опухоли головного мозга: общие принципы диагностики и лечения. Педиатр 2015;6(4):75-84. DOI: 10.17816/PED6475-84. [Trashkov A.P., Spirin A.L., Tsygan N.V. et al. Cerebral glial tumors: general principles of diagnostics and treatment. Pediatr $=$ Pediatrician 2015;6(4):75-84. (In Russ.)]. DOI: 10.17816/PED6475-84.

17. Дяченко А.А., Субботина А.В., Измайлов Т.Р. и др. Первичные злокачественные новообразования центральной нервной системы в Архангельской области: структура и динамика эпидемиологических показателей в 2000-2011 гг. Вестник Российского научного центра рентгенорадиологии 2013;(13-1):12. [Dyachenko A.A., Subbotina A.V., Izmajlov T.R. et al. Primary malignant tumors of the central nervous system in the Arkhangelsk Region, Russia: structure and dynamics of epidemiological indicators in 20002011. Vestnik Rossiyskogo nauchnogo tsentra rentgenoradiologii $=$ Vestnik of the Russian Scientific Center of Roentgenoradiology 2013;(13-1):12. (In Russ.)]. 
18. Hanif F., Muzaffar K., Perveen K. et al. Glioblastoma multiforme: a review of its epidemiology and pathogenesis through clinical presentation and treatment. Asian Pac J Cancer Prev 2017;18(1):3-9. DOI: 10.22034/APJCP.2017.18.1.3.

19. Измайлов Т.Р., Паньшин Г.А., Даценко П.В. Выбор режима фракционирования при лечении глиом высокой степени злокачественности (часть 1): возраст и степень злокачественности. Сибирский онкологический журнал. 2012;(2):11-7. [Izmaylov T.R.,

Panshin G.A., Datsenko P.V. Choice of fractionation regimen for treatment of high-grade gliomas (part 1): age and tumor grade. Sibirskiy onkologicheskiy zhurnal $=$ Siberian Journal of Oncology 2012;(2):11-7. (In Russ.)].

20. Kamiya-Matsuoka C., Gilbert M.R. Treating recurrent glioblastoma: an update. CNS Oncol 2015;4(2):91-104. DOI: $10.2217 / \mathrm{cns} .14 .5$.

21. Абсалямова О.В., Алешин В.А., Аникеева О.Ю. и др. Клинические рекомендации по диагностике и лечению больных с первичными опухолями головного мозга. М., 2014. 60 c. [Absalyamova O.V., Aleshin V.A., Anikeeva O.Yu. et al. Clinical recommendations for the diagnosis and treatment of patients with primary brain tumors. Moscow, 2014. 60 p. (In Russ.)].

22. Rice T., Lachance D.H., Molinaro A.M. et al. Understanding inherited genetic risk of adult glioma $-\mathrm{a}$ review. Neurooncol Pract 2016;3(1):10-6. DOI: $10.1093 /$ nop/npv026.

23. Baumgarten P., Quick-Weller J., Gessler F. et al. Pre- and early postoperative GFAP serum levels in glioma and brain metastases. J Neurooncol 2018;139(3):541-6. DOI: 10.1007/s11060-018-2898-1.

24. Van Bodegraven E.J., van Asperen J.V., Robe P. A.J., Hol E.M. Importance of GFAP isoform-specific analyses in astrocytoma. Glia 2019;67(8):1417-33. DOI: $10.1002 /$ glia.23594.

25. Cloughesy T.F., Cavenee W.K., Mischel P.S. Glioblastoma: from molecular pathology to targeted treatment. Annu Rev Pathol 2014;9:1-25. DOI: 10.1146/ annurev-pathol-011110-130324.

26. Agnihotri S., Burrell K.E., Wolf A. et al. Glioblastoma, a brief review of history, molecular genetics, animal models and novel therapeutic strategies. Arch Immunol Ther Exp (Warsz) 2013;61(1):25-41. DOI: $10.1007 / \mathrm{s} 00005-012-0203-0$.

27. Ohgaki H., Kleihues P. The definition of primary and secondary glioblastoma. Clin Cancer Res 2013;19(4):764-72. DOI: 10.1158/1078-0432.CCR-12-3002.

28. Malta T.M., de Souza C.F., Sabedot T.S. et al. Glioma $\mathrm{CpG}$ island methylator phenotype (G-CIMP): biological and clinical implications. Neuro Oncol 2018;20(5):608-20. DOI: $10.1093 /$ neuonc/nox183.
29. Hervey-Jumper S.L., Berger M.S. Maximizing safe resection of low- and high-grade glioma. J Neurooncol 2016;130(2):269-82. DOI: 10.1007/s11060-016-2110-4.

30. Chen W.J., He D.S., Tang R.X. et al. $\mathrm{Ki}-67$ is a valuable prognostic factor in gliomas: evidence from a systematic review and meta-analysis. Asian Pac J Cancer Prev 2015;16(2):411-20. DOI: 10.7314/apjcp.2015.16.2.411.

31. Yan H., Parsons D.W., Jin G. et al. IDH1 and IDH2 mutations in gliomas. N Engl J Med 2009;360(8):765-73. DOI: 10.1056/NEJMoa0808710.

32. Snyder L.A., Wolf A.B., Oppenlander M.E. et al. The impact of extent of resection on malignant transformation of pure oligodendrogliomas. J Neurosurg 2014;120(2):309-14.

DOI: $10.3171 / 2013.10 . J N S 13368$.

33. Ferguson S., Lesniak M.S. Percival Bailey and the classification of brain tumors. Neurosurg Focus 2005;18(4):e7. DOI: $10.3171 /$ foc. 2005.18 .4 .8 .

34. Huse J.T., Diamond E.L., Wang L., Rosenblum M.K. Mixed glioma with molecular features of composite oligodendroglioma and astrocytoma: a true "oligoastrocytoma"? Acta Neuropathol 2015;129(1):151-3. DOI: 10.1007/s00401-014-1359-y.

35. Elaimy A.L., Mackay A.R., Lamoreaux W.T. et al. Clinical outcomes of Gamma Knife radiosurgery in the salvage treatment of patients with recurrent high-grade glioma. World Neurosurg 2013;80(6):872-8. DOI: 10.1016/j.wneu.2013.02.030.

36. Nayak L., Reardon D.A. High-grade gliomas. Continuum (Minneap Minn) 2017;23(6, Neuro-oncology):1548-63. DOI: $10.1212 /$ con. 0000000000000554 .

37. Stupp R., Roila F. Malignant glioma: ESMO clinical recommendations for diagnosis, treatment and follow-up. Ann Oncol 2009;20 Suppl 4:126-8. DOI: 10.1093/annonc/mdp151.

38. Hanif F., Muzaffar K., Perveen K. et al. Glioblastoma multiforme: a review of its epidemiology and pathogenesis through clinical presentation and treatment. Asian Pac J Cancer Prev 2017;18(1):3-9. DOI: 10.22034/APJCP.2017.18.1.3.

39. Davis M.E. Glioblastoma: overview of disease and treatment. Clin J Oncol Nurs 2016;20(5 Suppl):S2-8. DOI: 10.1188/16.CJON.S1.2-8.

40. Klein J.P., Dietrich J. Neuroradiologic pearls for neuro-oncology. Continuum (Minneap Minn) 2017;23(6, Neurooncology):1619-34. DOI: $10.1212 /$ con.0000000000000543.

41. Mihovilovic M.I., Kertels O., Hänscheid H. et al. O- $\left(2-\left({ }^{18} \mathrm{~F}\right)\right.$ fluoroethyl)-L-tyrosine PET for the differentiation of tumour recurrence from late pseudoprogression in glioblastoma. J Neurol Neurosurg Psychiatry 2019;90(2):238-9. DOI: 10.1136/jnnp-2017-317155.
42. Трофимова Т.Н., Олюшин В.Е., Порсаев А.И. и др. Комплексная оценка радикальности удаления супратенториальных глиом. Лучевая диагностика и терапия 2015;(2):54-62.

[Trofimova T.N., Olyushin V.E., Porsaev A.I. et al. Complex assesement of radical resection of supratentorial gliomas. Luchevaya diagnostika i terapiya $=$ Diagnostic Radiology and Radiotherapy 2015;(2):54-62. (In Russ.)].

43. Токарев А.С., Рак В.А., Евдокимова О.Л. и др. Оценка ранних результатов радиохирургического лечения рецидивирующих глиобластом головного мозга с использованием мультимодальной нейровизуализации. Русский медицинский журнал 2017;25(16):1200.

[Tokarev A.S., Rak V.A., Evdokimova O.L. et al. Evaluation of early results of radiosurgical treatment of recurrent brain glioblastomas using multimodal neuroimaging. Russkiy medicinskiy zhurnal $=$ Russian Medical Journal 2017;25(16):1200. (In Russ.)].

44. Токарев А.С., Чувилин С.А., Степанов В.Н., Рак В.А. Роль функциональной магнитно-резонансной томографии при удалении опухолей в функционально значимых зонах головного мозга. Нейрохирургия 2017;(3):64-9.

[Tokarev A.S., Chuvilin S.A., Stepanov V.N., Rak V.A. Role of functional magnetic resonance imaging during removal of tumors in brain eloquent areas. Neyrokhirurgiya $=$ Russian Journal of Neurosurgery 2017;(3):64-9. (In Russ.)].

45. Hassani D.F., Melhaoui A., Dif Y. et al. Integration of three-dimensional magnetic resonance imaging spectroscopy with the Leksell GammaPlan radiosurgical planning station for the treatment of brain tumors. Cureus 2019;11(10):e5946. DOI: 10.7759 /cureus.5946.

46. Arora G., Sharma P., Sharma A. et al. ${ }^{99 \mathrm{~m}} \mathrm{Tc}$-methionine hybrid SPECT/CT for detection of recurrent glioma: comparison with 18F-FDG PET/CT and contrastenhanced MRI. Clin Nucl Med 2018;43(5):e132-8.

DOI: 10.1097/RLU.0000000000002036.

47. Butenschön V.M., Ille S., Sollmann N. et al. Cost-effectiveness of preoperative motor mapping with navigated transcranial magnetic brain stimulation in patients with high-grade glioma. Neurosurg Focus 2018;44(6):E18. DOI: $10.3171 / 2018.3$.FOCUS1830.

48. Порсаев А.И., Олюшин В.Е., Трофимова Т.Н. и др. Сравнительный анализ информативности магнитно-резонансной томографии и УЗИ при оценке степени радикальности удаления супратенториальных глиом в раннем послеоперационном периоде. Российский нейрохирургический журнал им. А.Л. Поленова 2013;5(4):33-39. [Porsaev A.I., Olushin V.E., Trofimova T.N. A comparative analysis 
of informational content of magnetic resonance tomography and ultrasound in assessing the extent of radical removal of supratentorial gliomas in the early postoperative period. Rossiyskiy neyrokhirurgicheskiy zhurnal im. A.L. Polenova = Russian Neurosurgical Journal n. a. A.L. Polenov 2013;5(4):33-39. (In Russ.)].

49. Lawrence Y.R., Wang M., Dicker A.P. et al. Early toxicity predicts long-term survival in high-grade glioma. Br J Cancer 2011;104(9):1365-71. DOI: $10.1038 /$ bjc.2011.123.

50. D'Amico R.S., Englander Z.K., Canoll P., Bruce J.N. Extent of resection in glioma a review of the cutting edge. World Neurosurg 2017;103:538-49. DOI: 10.1016/j.wneu.2017.04.041.

51. Kageji T., Nagahiro S., Uyama S. et al. Histopathological findings in autopsied glioblastoma patients treated by mixed neutron beam BNCT. J Neurooncol 2004;68(1):25-32. DOI: 10.1023/ b:neon.0000024725.31515.22.

52. Lacroix M., Abi-Said D., Fourney D.R et al. A multivariate analysis of 416 patients with glioblastoma multiforme: prognosis, extent of resection, and survival. J Neurosurg 2001;95(2):190-8. DOI: $10.3171 /$ jns.2001.95.2.0190.

53. de Leeuw C.N., Vogelbaum M.A. Supratotal resection in glioma: a systematic review. Neuro Oncol 2019;21(2):179-88 DOI: $10.1093 /$ neuonc/noy166.

54. Grabowski M.M., Recinos P.F., Nowacki A.S. et al. Residual tumor volume versus extent of resection: predictors of survival after surgery for glioblastoma. J Neurosurg 2014;121(5):1115-23. DOI: $10.3171 / 2014.7 . J N S 132449$.

55. Sanai N., Berger M.S. Surgical oncology for gliomas: the state of the art. Nat Rev Clin Oncol 2018;15(2):112-25. DOI: $10.1038 /$ nrclinonc.2017.171.

56. Chaichana K.L., Jusue-Torres I., Navarro-Ramirez R. et al. Establishing percent resection and residual volume thresholds affecting survival and recurrence for patients with newly diagnosed intracranial glioblastoma. Neuro Oncol 2014;16(1):113-22. DOI: $10.1093 /$ neuonc/not 137 .

57. Weller M., van den Bent M., Hopkins K et al. EANO guideline for the diagnosis and treatment of anaplastic gliomas and glioblastoma. Lancet Oncol 2014;15(9):e395-403. DOI: $10.1016 / \mathrm{S} 1470-2045(14) 70011-7$.

58. Kyritsis A.P., Levin V.A. An algorithm for chemotherapy treatment of recurrent glioma patients after temozolomide failure in the general oncology setting. Cancer Chemother Pharmacol 2011;67(5):971-83. DOI: $10.1007 / \mathrm{s} 00280-011-1617-9$.

59. Taal W., Bromberg J.E., van den Bent M.J. Chemotherapy in glioma. CNS Oncol 2015;4(3):179-92. DOI: $10.2217 / \mathrm{cns} .15 .2$.
60. Van den Bent M.J. Interobserver variation of the histopathological diagnosis in clinical trials on glioma: a clinician's perspective. Acta Neuropathol 2010;120(3):297-304. DOI: $10.1007 / \mathrm{s} 00401-010-0725-7$.

61. Yung W.K., Albright R.E., Olson J. et al. A phase II study of temozolomide $v s$. procarbazine in patients with glioblastoma multiforme at first relapse. Br J Cancer 2000;83(5):588-93.

DOI: $10.1054 /$ bjoc. 2000.1316 .

62. Stupp R., Hegi M.E., Mason W.P. et al. Effects of radiotherapy with concomitant and adjuvant temozolomide versus radiotherapy alone on survival in glioblastoma in a randomised phase III study: 5-year analysis of the EORTCNCIC trial. Lancet Oncol 2009;10(5):459-66. DOI: 10.1016/s1470-2045(09)70025-7.

63. Perry J.R., Rizek P., Cashman R. et al. Temozolomide rechallenge in recurrent malignant glioma by using a continuous temozolomide schedule: the "rescue" approach. Cancer 2008;113(8):2152-7. DOI: $10.1002 /$ cncr.23813.

64. Shin J.Y., Diaz A.Z. Utilization and impact of adjuvant therapy in anaplastic oligodendroglioma: an analysis on 1692 patients. J Neurooncol 2016;129(3):567-75. DOI: 10.1007/s11060-016-2212-z.

65. Brada M., Stenning S., Gabe R. et al. Temozolomide versus procarbazine, lomustine, and vincristine in recurrent high-grade glioma. J Clin Oncol 2010;28(30):4601-8. DOI: 10.1200/JCO.2009.27.1932.

66. Van Den Bent M.J., Erdem-Eraslan L., Idbaih A. et al. MGMT-STP27 methylation status as predictive marker for response to $\mathrm{PCV}$ in anaplastic oligodendrogliomas and oligoastrocytomas. A report from EORTC study 26951. Clin Cancer Res 2013;19(19):5513-22. DOI: 10.1158/1078-0432.CCR-13-1157.

67. Ashby L.S., Smith K.A., Stea B. Gliadel wafer implantation combined with standard radiotherapy and concurrent followed by adjuvant temozolomide for treatment of newly diagnosed high-grade glioma: a systematic literature review. World J Surg Oncol 2016;14(1):225. DOI: $10.1186 / \mathrm{s} 12957-016-0975-5$.

68. Izquierdo C., Joubert B., Ducray F. Anaplastic gliomas in adults: an update. Curr Opin Oncol 2017;29(6):434-42. DOI: $10.1097 /$ cco.0000000000000409.

69. Wheeler C.J. Dendritic cell vaccines to combat glioblastoma. Expert Rev Neuroth 2010;10(4):483-6. DOI: $10.1586 /$ ern.10.26.

70. Carrillo J.A., Hsu F.P.K., Delashaw J., Bota D.A. Efficacy and safety of bevacizumab and etoposide combination in patients with recurrent malignant gliomas who have failed bevacizumab. Rev Health Care 2014;5(1):23-32. DOI: $10.7175 /$ rhc.v5i1.668.
71. Chen R., Cohen A.L., Colman H. Targeted therapeutics in patients with high-grade gliomas: past, present, and future. Curr Treat Options Oncol 2016;17(8):42. DOI: 10.1007/s11864-016-0418-0.

72. Ameratunga M., Pavlakis N., Wheeler H. et al. Anti-angiogenic therapy for highgrade glioma. Cochrane Database Syst Rev 2018;11(11):CD008218. DOI: 10.1002/14651858.CD008218.pub4.

73. Batchelor T.T., Mulholland P., Neyns B. et al. Phase III randomized trial comparing the efficacy of cediranib as monotherapy, and in combination with lomustine, versus lomustine alone in patients with recurrent glioblastoma. J Clin Oncol 2013;31(26):3212-8. DOI: 10.1200/JCO.2012.47.2464.

74. Bokstein F., Blumenthal D.T., Corn B.W. et al. Stereotactic radiosurgery (SRS) in high-grade glioma: judicious selection of small target volumes improves results. J Neurooncol. 2016;126(3):551-7. DOI: $10.1007 / \mathrm{s} 11060-015-1997-5$.

75. Clark G.M., McDonald A.M., Nabors L.B. et al. Hypofractionated stereotactic radiosurgery with concurrent bevacizumab for recurrent malignant gliomas: the University of Alabama at Birmingham experience. Neurooncol Pract. 2014;1(4):172-177. PMID: 26034629. DOI: $10.1093 /$ nop/npu028.

76. Chinot O.L., Wick W., Mason W. et al. Bevacizumab plus radiotherapy-temozolomide for newly diagnosed glioblastoma. N Engl J Med 2014;370(8):709-22.

77. Field K.M., King M.T., Simes J. et al. Health-related quality of life outcomes from CABARET: a randomized phase 2 trial of carboplatin and bevacizumab in recurrent glioblastoma. J Neurooncol 2017;133(3):623-31. DOI: $10.1007 / \mathrm{s} 11060-017-2479-8$.

78. Andronesi O.C., Loebel F., Bogner W. et al. Treatment Response Assessment in IDH-mutant glioma patients by noninvasive $3 \mathrm{D}$ functional spectroscopic mapping of 2-hydroxyglutarate. Clin Cancer Res 2016;22(7):1632-41. DOI: 10.1158/1078-0432.CCR-15-0656.

79. Johnson B.E., Mazor T., Hong C. et al. Mutational analysis reveals the origin and therapy-driven evolution of recurrent glioma. Science 2014;343(6167):189-93. DOI: $10.1126 /$ science. 1239947.

80. Picca A., Berzero G., Di Stefano A.L., Sanson M. The clinical use of IDH1 and IDH2 mutations in gliomas. Expert Rev Mol Diagn 2018;18(12):1041-51. DOI: 10.1080/14737159.2018.1548935.

81. Климанов В.А. Радиобиологическое и дозиметрическое планирование лучевой и радионуклидной терапии. Ч. 2. Лучевая терапия пучками протонов, ионов, нейтронов и пучками с модулированной интенсивностью, стереотаксис, брахитерапия, радионуклидная терапия, оптимизация, гарантия. М.: НИЯУ МИФИ, 2011. 604 с. 
[Klimanov V.A. Radiobiological and dosimetric planning of radiation and radionuclide therapy. Part 2 . Radiation therapy with proton, ion, neutron beams and beams with modulated intensity, stereotaxis, brachytherapy, radionuclide therapy, optimization, guarantee. Moscow: NRNU MEPHI, 2011. 604 p. (In Russ.)].

82. Bergonie J., Tribondeau L. De quelques resultats de la radiotherapie et essai de fixation d'une technique rationnelle. Comptes Rendus des Seances de l'Academie des Sciences. 1906;143:983-5.
83. Dalesandro M.F., Andre J.B. Posttreatment Evaluation of Brain Gliomas. Neuroimaging Clin N Am 2016;26(4):581-99. DOI: $10.1016 /$ j.nic. 2016.06.007.

84. Sourati A., Ameri A., Malekzadeh M Radiation brain injury. In: Acute side effects radiation therapy. Springer, 2017. Pp. 27-37.

DOI: 10.1007/978-3-319-55950-6_3.

85. Malmström A., Grønberg B.H., Marosi C. et al. Temozolomide versus standard 6-week radiotherapy versus hypo- fractionated radiotherapy in patients older than 60 years with glioblastoma: the Nordic randomised, phase 3 trial. Lancet Oncol 2012;13(9): 916-26. DOI: $10.1016 / \mathrm{s} 1470-2045(12) 70265-6$.

86. Warren L.E.G., Bussiére M.R., Shih H.A. Radiation therapy for malignant gliomas: current options. In: Malignant brain tumors.

Ed. by J.M. Gunel, J.M. Piepmeier, J.M. Baehring. Springer, 2016.

Pp. 217-231.

DOI: 10.1007/978-3-319-49864-5 14

\section{Вклад авторов}

К.В. Грецких, А.С. Токарев: обзор публикаций по теме статьи, написание текста статьи. Authors' contributions

K.V. Gretskikh, A.S. Tokarev: reviewing of publications on the article's theme, article writing.

ORCID авторов / ORCID of authors

K.В. Грецких / K.V. Gretskikh: https://orcid.org/0000-0003-1042-0837

A.C. Токарев / A.S. Tokarev: https://orcid.org / 0000-0002-8415-5602

Конфликт интересов. Авторы заявляют об отсутствии конфликта интересов.

Conflict of interest. The authors declare no conflict of interest.

Финансирование. Работа выполнена без спонсорской поддержки.

Financing. The work was performed without external funding. 